\title{
Prediction of Jet Noise Shielding
}

\author{
Dimitri Papamoschou* \\ University of California, Irvine, CA 92697, USA
}

\begin{abstract}
This study is motivated by the development of aircraft that use jet noise shielding by the airframe. Current methods to predict shielding from aircraft surfaces rely on formulae developed for diffraction of sound from omnidirectional point sources. However, the jet noise source is distributed and directional, thus requiring a different approach. In this study, the jet noise source is modeled as the incoherent superposition of a wavepacket and a monopole. Source parameterization and application of minimization methods are used to match the directivity of the far-field sound at a given frequency. The resulting incident field is then used to compute the diffraction problem using the Boundary Element Method. Reasonable agreement is found with experimental data, suggesting the applicability of the wavepacket ansatz in modeling the jet noise source.
\end{abstract}

\section{Introduction}

This study is motivated by the development of ultra-quiet advanced aircraft that use jet noise shielding by the airframe. In such aircraft the engines would be mounted over the wing (OTW). Significant experimental research on OTW conventional and short-takeoff airplanes occurred in the 1970s (Ref.1 for example). Important trends were established for the changes in the spectrum of acoustic emission versus shield parameters. However, the concept found very little commercial application at that time and research dried up.

The advent of the Hybrid Wing-Body (HWB) airplane, with the engines mounted over the wing, has reinvigorated the OTW concept for jet noise shielding. The HWB design allows sufficient planform area for shielding of both the forward-emitting turbomachinery sources and the aft-emitting jet noise sources. To properly integrate the engine with the airframe for jet noise shielding, physics-based predictive tools must be developed. The challenge is that jet noise is a distributed and directive source, whose exact nature remains under investigation. The current "state of the art" in empirical prediction of jet noise shielding involves approximating the noise source as a small number of discrete sources ${ }^{2}$ combined with insertion loss formulas developed for barrier insertion losses of sound from point sources. The insertion loss formula is based on Maekawa's experiments ${ }^{3}$ and involves only the Fresnel number. The current state of the art is thus inadequate because jet noise is a distributed directive source while the barrier-insertion relations were developed for omnidirectional point sources. Development of reliable, physics-based predictive tools for jet noise shielding is inextricably connected to properly describing the jet noise source. Given the complexity of sound generation by turbulent mixing, one must resort to simplified models that retain some of the essential physics - such as the wavepacket model for noise generation from large-scale structures. The intent is to develop predictive methodologies that will be used in the nextgeneration ANOPP tools for aircraft noise. The tools must be useful in the sense that they should not require tremendous amount of computational resources.

This paper expands initial efforts in modeling of jet noise shielding ${ }^{4}$ with more accurate and efficient implementations of the noise source model and diffraction methods. In addition, the inappropriateness of using point source approximations for jet noise vis-à-vis diffraction is explained on a theoretical basis. To appreciate the nature of jet noise diffraction, it is helpful to examine some acoustic data involving simple jets and shields. Figure 1 shows the basic setup for the experiments and the computations in this paper. Figure 2 shows narrowband sound pressure level spectra for one of the configurations tested. The

\footnotetext{
*Professor, Department of Mechanical and Aerospace Engineering, dpapamos@uci.edu, AIAA Fellow.
} 
spectra are plotted against Strouhal number $S r=f D_{j} / U_{j}$. With increasing polar angle $\theta$ from the jet axis shielding becomes more pronounced for $S r \geq 0.5$ but there is substantial noise excess for $S r<0.5$. This is consistent with trends observed in previous works ${ }^{1}$, which attributed the excess noise to jet scrubbing the shielding surface. However for the experiment shown in Fig.1 it was verified, using Pitot surveys, that the jet did not contact the shielding plate. The excess noise therefore is not necessarily connected to scrubbing and may also be caused by the jet noise source and its interaction with the surface. Any physical model should be able to predict not only the noise suppression but also the noise excess created by the boundary.

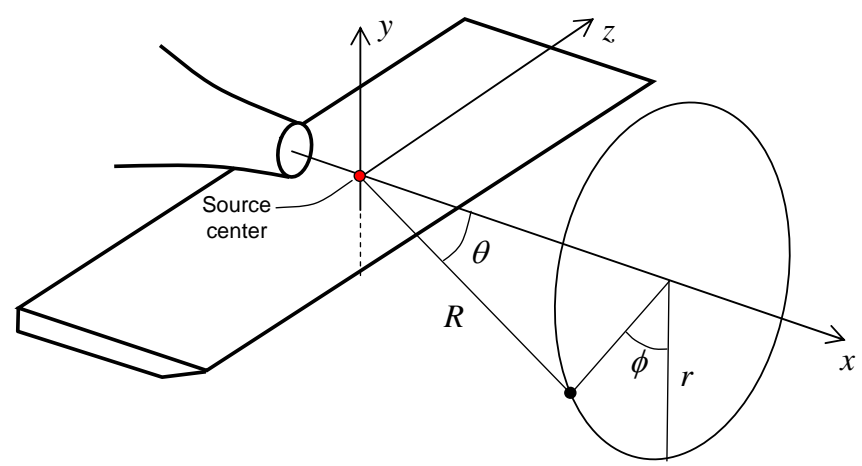

Fig. 1 Jet and boundary configuration considered in this study.
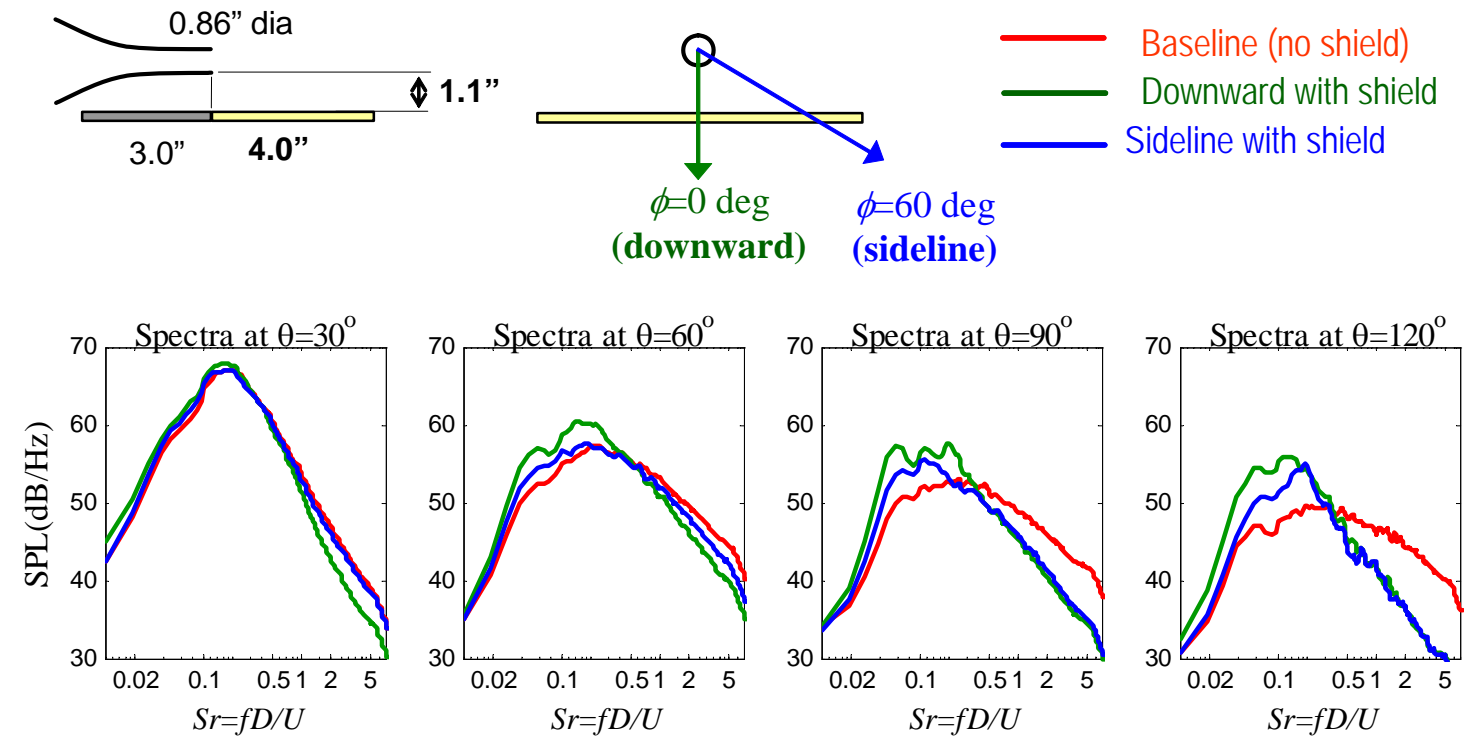

Fig. 2 Experimental spectra of noise shielding from a Mach 0.9 cold jet. The span of the rectangular shield was 24 inches. 

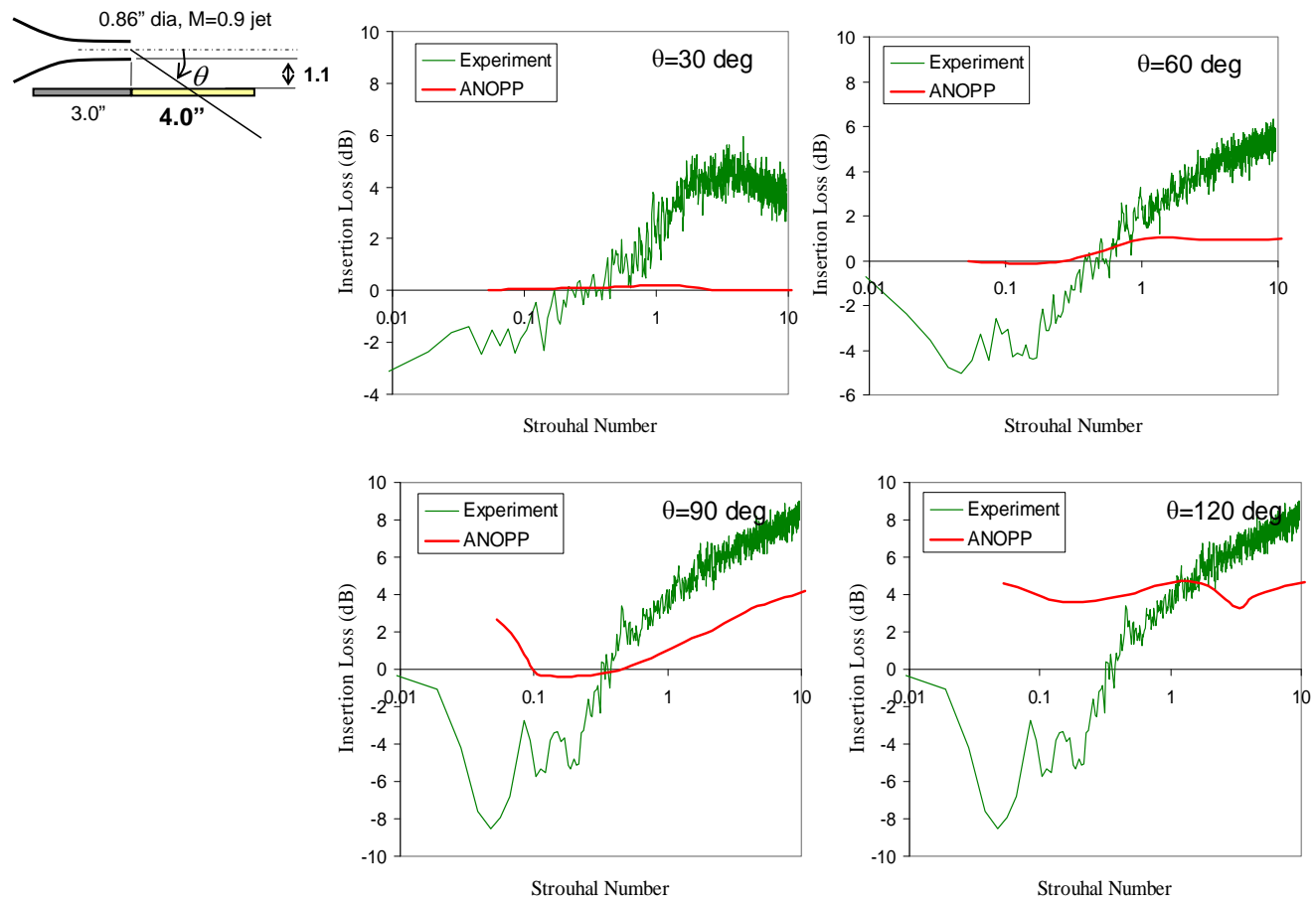

Fig. 3 Insertion loss for configuration shown on top left. Experimental measurements and ANOPP-based predictions are compared at difference polar angles.

\section{Assessment of Point Source Approximation of Jet Noise}

The first step in our investigation was to assess the accuracy with which existing tools in ANOPP could predict jet noise shielding. The tools are the ST2JET module ${ }^{2}$ and Maekawa's insertion-loss formula ${ }^{3}$ used in the WING module. The Maekawa relation is based on geometric acoustics from omni-directional point sources. The ST2JET module simplifies the jet noise source a number of independent point sources. The results of the ANOPP-based shielding prediction were compared to experimental data from past works and from subscale tests in the UCI lab. The comparisons were not encouraging. As shown in Fig. 3, ANOPP-based predictions of insertion loss are in strong disagreement with experiments. The basic reasons for the disagreement are that (a) the noise source is much more complex than a collection of independent point sources and (b) the shielding tool used (Maekawa's formula) is inappropriate for a complex source like the jet.

Let us consider the effect of the directivity of the jet noise. Here we are concerned with the acoustic near field of the jet, because diffraction occurs in the near field. Can we make the approximation that, in the near field, sound of a directional source comes from a point? To answer this question, we note that an acoustic pressure with directivity in polar angle $\theta$ can be reconstructed in terms of Legendre polynomials:

$$
p(R, \theta, t)=\sum_{m=0}^{\infty} A_{m} P_{m}(\cos \theta) h_{m}(k R) e^{i \omega t}
$$

where $P_{m}$ are the Legendre polynomials, $h_{m}$ are spherical Hankel functions, and $k=\omega / a_{\infty}$ is the wavenumber. As $k r \rightarrow \infty$, the spherical Hankel function is approximated by

$$
h_{m}(k R)=i^{-(m+1)} \frac{e^{i k R}}{k R}
$$

and the acoustic pressure takes the form 


$$
p(R, \theta, t)=\sum_{m=0}^{\infty} A_{m} P_{m}(\cos \theta) i^{-(m+1)} \frac{e^{i k R}}{k R} e^{i \omega t}=\frac{e^{i(k R-\omega t)}}{R} \sum_{m=0}^{\infty} \frac{A_{m}}{k} P_{m}(\cos \theta) i^{-(m+1)}
$$

Equation (2) represents a directive pressure field that comes from a point and can be written as

$$
p(R, \theta, t)=\frac{e^{i(k R-\omega t)}}{R} \Psi(\theta)
$$

The first term on the right-hand side is sound emission from a monopole, and the second term $\Psi(\theta)$ is the polar directivity of the far acoustic field. In the far-field formulation of Eq.3, the directivity $\Psi$ is a real function. If we apply the formulation of Eq.3 in the near field, we realize that $\Psi$ becomes complex and its distribution is different from that in the far-field. This will be illustrated by examples to follow. Comparing Eqs (1) and (2), it become evident that the validity of the point-source approximation depends on how close the function

$$
g_{m}(k R)=i^{m+1} \frac{k r}{e^{i k r}} h_{m}(k R)
$$

approaches unity on the complex plane, i.e., how close it is to $1+0 i$. To quantify the error in the pointsource approximation, we look at the departure of $g_{m}$ from unity:

$$
\text { Departure }=\sqrt{\left(\operatorname{Re}\left\{g_{m}\right\}-1\right)^{2}+\left(\operatorname{Im}\left\{g_{m}\right\}\right)^{2}}
$$

To accurately reconstruct a typical jet noise field using Eq.1, our experience indicates that we need at least five Legendre terms. Figure 4 plots the departure from unity of the function $g_{5}$ versus distance and frequency for a full-scale application. This figure illustrates the error in the point-source approximation for a directional acoustic field. For frequencies of relevance to aircraft certification, the error is unacceptably high (more than 50\%) for typical distances associated with shielding (a few meters). This addresses only one complexity of the jet noise source. The other major complication is that the source has a finite spatial coherence.

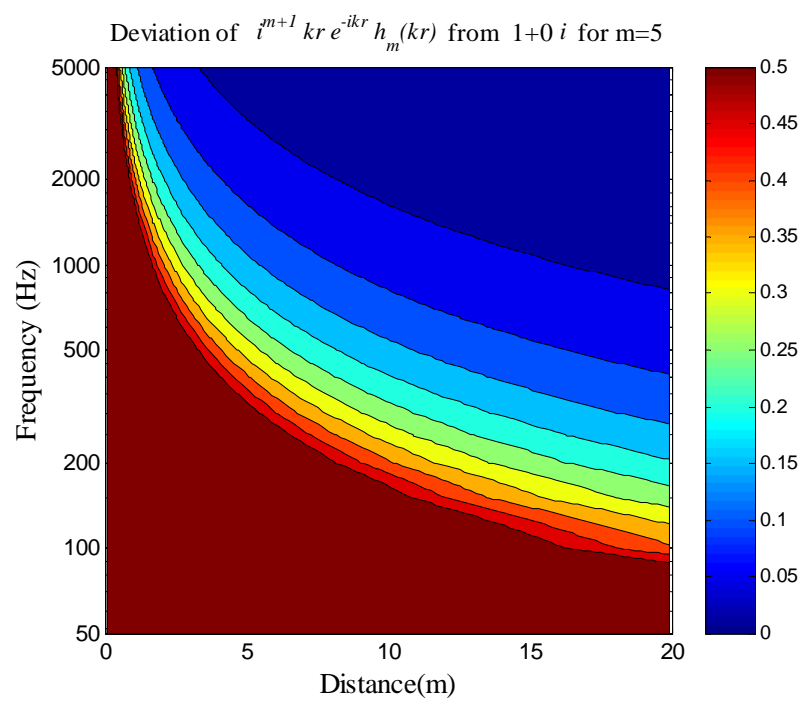

Fig. 4 Error in approximating a directional acoustic field as coming from a point. Brown region indicates more than $50 \%$ error.

\section{Jet Noise Source Model}

It is generally agreed that sound emission in the aft direction, at shallow angles to the jet axis, is caused by large-scale turbulent structures while noise emitted at large angles to the jet axis is caused by fine- 
scale turbulent motions ${ }^{5}$. The former is highly directional, while the latter radiates uniformly. The largescale structures can be modeled as instability waves that grow and then decay with axial distance. This is model based on the foundational works by Tam and Burton ${ }^{6}$, Crighton and Huerre ${ }^{7}$, and Avital et al. ${ }^{8}$, with recent contributions by Morris ${ }^{9}$. The jet is replaced by a cylinder $r=r_{0}$ on which we prescribe the pressure perturbation

$$
p_{w}\left(m, r_{0}, x, \varphi, t\right)=p_{0}(x) e^{-i \omega t+i m \phi}
$$

where $m$ denotes the azimuthal mode and $\phi$ is the azimuthal angle. Denoting the spatial Fourier transform of $p_{0}(x)$ as $\hat{p}_{0}(k)$, the solution for $r \geq r_{0}$ is ${ }^{6,9}$

$$
\begin{gathered}
p_{w}(m, r, x, \varphi, t)=\frac{1}{2 \pi} e^{-i \omega t+i m \phi} \int_{-\infty}^{\infty} \hat{p}_{0}(k) \frac{H_{m}^{(1)}(\lambda r)}{H_{m}^{(1)}\left(\lambda r_{0}\right)} e^{i k x} d k \\
\lambda=\left[\left(\frac{\omega}{a_{\infty}}\right)^{2}-k^{2}\right]^{1 / 2}, \quad-\frac{\pi}{2}<\arg (\lambda)<\frac{\pi}{2}
\end{gathered}
$$

Equation 5 is the exact solution to the linearized problem, valid everywhere for $r \geq r_{0}$. Once the wavepacket shape $p_{0}(x)$ is determined (through the minimization scheme explained in the next section), Eq. 5 is used to obtain the incident field $p_{i}$ on the object surface and at the field points. The actual computation of Eq.5 involves taking the forward FFT of $p_{0}(x)$ to obtain $\hat{p}_{0}(k)$, then the inverse FFT of $\hat{p}_{0}(k)$ multiplied by the Hankel functions. An important aspect of the pressure field generated by the wavepacket is that it has a radiative (supersonic) component and a decaying (subsonic) component. It is useful to separate the two in order to gain insights into the mechanisms of diffraction. The decaying component involves phase speeds that are subsonic, $|\omega / k|<a_{\infty}$ or $|k|>\omega / a_{\infty}$ :

$$
p_{w, \text { sub }}(m, r, x, \varphi, t)=\frac{1}{2 \pi} e^{-i \omega t+i m \phi} \int_{|k|>\omega / a_{\infty}} \hat{p}_{0}(k) \frac{H_{m}^{(1)}(\lambda r)}{H_{m}^{(1)}\left(\lambda r_{0}\right)} e^{i k x} d k
$$

The radiating component involves phase speeds that are sonic or supersonic, $|k| \leq \omega / a_{\infty}$ :

$$
p_{w, \text { sup }}(m, r, x, \varphi, t)=\frac{1}{2 \pi} e^{-i \omega t+i m \phi} \int_{-\omega / a_{\infty}}^{\omega / a_{\infty}} \hat{p}_{0}(k) \frac{H_{m}^{(1)}(\lambda r)}{H_{m}^{(1)}\left(\lambda r_{0}\right)} e^{i k x} d k
$$

From Eq.5b, using the convolution property of the Fourier transform, we obtain an expression for the part of the $p_{0}(x)$ that radiates to the far field:

$$
p_{0, \text { sup }}(x)=\frac{1}{\pi} \int_{-\infty}^{\infty} \frac{p_{0}(\xi)}{x-\xi} \sin \left[\frac{\omega}{a_{\infty}}(x-\xi)\right] d \xi
$$

This is relevant to the axial alignment of the wavepacket based on far-field noise source location measurements, discussed in the next section. The far-field approximation of Eq.5 is ${ }^{9}$

$$
p_{w, f a r}(m, R, \theta, \varphi, t)=-\frac{i}{\pi R} \frac{\hat{p}_{0}\left(\frac{\omega}{a_{\infty}} \cos \theta\right)}{H_{m}^{(1)}\left(\frac{\omega}{a_{\infty}} r_{0} \sin \theta\right)} e^{i \omega R / a_{\infty}} e^{-i \omega t+i m \phi}
$$

where $R$ is the distance of the observer from the origin and $\theta$ is the polar angle from the downstream wavepacket (jet) axis. The modulus squared of Eq.7 yields the modeled autospectrum of the far-field pressure: 


$$
S_{w, f a r}(m, R, \theta, \omega)=\frac{1}{(\pi R)^{2}}\left|\frac{\hat{p}_{0}\left(\frac{\omega}{a_{\infty}} \cos \theta\right)}{H_{m}^{(1)}\left(\frac{\omega}{a_{\infty}} r_{0} \sin \theta\right)}\right|^{2}
$$

For fixed frequency $\omega$, Eq.8 gives the polar intensity distribution. The simplicity of the far-field solution lends itself to the minimization process for determining the wavepacket shape, wherein Eq. 8 needs to be evaluated hundreds of times.

The wavepacket model by itself is unable to capture the directivity of jet noise at large polar angles from the jet axis. It is therefore necessary to combine it with an additional noise source that has omnidirectional character. This is in line with the two-source model of jet noise ${ }^{5}$. Here we use a simple point source (monopole) whose incident field is given by

$$
p_{p}(R, t)=\frac{Q}{4 \pi R} e^{-i \omega t+i k R}
$$

where $Q$ denotes the monopole strength. The wavepacket and monopole sources are assumed to be uncorrelated to each other, so the autospectrum of the combined field is the summation of the individual autospectra. In the far field, therefore, the modeled autospectrum is

$$
S_{\text {mod }}(m, R, \theta, \omega)=\frac{1}{(\pi R)^{2}}\left|\frac{\hat{p}_{0}\left(\frac{\omega}{a_{\infty}} \cos \theta\right)}{H_{m}^{(1)}\left(\frac{\omega}{a_{\infty}} r_{0} \sin \theta\right)}\right|^{2}+\frac{Q^{2}}{16 \pi^{2} R^{2}}
$$

\section{A. General approach}

\section{Model Parameterization}

The current philosophy of the wavepacket parameterization is determination of the noise source using experimental data that are either already available or not too difficult to obtain. It is noted that highly sophisticated experiments, using elaborate near-field microphone arrays, have been applied to the study of jet noise and specifically the detection of wavepackets (Ref. 10, for example). These experiments have yielded valuable insight into the physics of jet noise; however, were done on a limited set of nozzle configurations. It is not reasonable to expect that such elaborate data will be widely available for the type of predictive tools being developed here. In this study the parameterization is based on the availability of experimental far-field sound pressure level (SPL) spectra, and knowledge of the peak noise source location versus frequency.

\section{B. Mathematical procedure}

There are infinite choices for the wavepacket shape $p_{0}(x)$ in Eq.4, so one needs to narrow down the scope to a set of generic functions that can be described in terms of a finite number of parameters. Upon selecting a functional form, the wavepacket shape for given frequency $\omega$ and azimuthal mode $m$ can be expressed as

$$
p_{0}\left(x, A_{k}\right)
$$

where $A_{k}, k=1, . ., K-1$, is a vector consisting of $K-1$ parameters that define the wavepacket shape. The $K^{\text {th }}$ parameter is reserved for the monopole strength $Q$. The parameterization is conducted for a fixed frequency $\omega$ and distance $R$. Given a functional form for $p_{0}(x)$ and a parameter $A_{k}$, we obtain the modeled intensity distribution $S_{\text {mod }}\left(\theta, m, A_{k}\right)$ from Eq. 10 . The idea is then to select the parameter vector $A_{k}$ in a way that minimizes the difference between the modeled intensity distribution $S_{\bmod }\left(\theta, m, A_{k}\right)$ and the 
experimental intensity distribution $S_{\text {exp }}(\theta)$. Realizing that we are interested in matching the shape (directivity) of the polar intensity distribution, and not so much its absolute value, we deal with the normalized values of the modeled and experimental intensities:

$$
\begin{aligned}
& S_{\text {mod }}^{*}\left(\theta, m, A_{k}\right)=\frac{S_{\text {mod }}\left(\theta, m, A_{k}\right)}{S_{\text {mod,max }}\left(m, A_{k}\right)} \\
& S_{\text {exp }}^{*}(\theta)=\frac{S_{\text {exp }}(\theta)}{S_{\text {exp,max }}}
\end{aligned}
$$

where max denotes the peak value of the polar distribution. The above normalization eliminates the amplitude constant from the minimization process. Once the shape is matched, the absolute levels can be matched through a trivial adjustment of the amplitude.

For a given jet flow, the experimental intensity distribution (autospectrum) is known at discrete polar angles $\theta_{j}, j=1, . ., J$. We construct a cost function based on the relative difference between the modeled and experimental intensity distributions at all the measurement polar angles,

$$
F\left(A_{k}\right)=\frac{1}{J} \sum_{j=1}^{J}\left|\frac{S_{\text {exp }}^{*}\left(\theta_{j}\right)-S_{\text {mod }}^{*}\left(m, A_{k}, \theta_{j}\right)}{S_{\exp }^{*}\left(\theta_{j}\right)}\right|^{2}
$$

We then seek determination of $A_{k}$ that minimizes the cost function. However, indiscriminate use of Eq.12 can easily lead to non-physical outcomes for $A_{k}$. It is typically necessary to constraint key parameters of the problem to ranges that are known a priori. Supposing that we want to constraint the parameter $A_{n}$ to be near a target value $A_{n \text {, target }}$, we add a corresponding penalty to the cost function:

$$
F\left(A_{k}\right)=\frac{1}{J} \sum_{j=1}^{J}\left|\frac{S_{\exp }^{*}\left(\theta_{j}\right)-S_{\text {mod }}^{*}\left(m, A_{k}, \theta_{j}\right)}{S_{\exp }^{*}\left(\theta_{j}\right)}\right|^{2}+C\left(A_{n}-A_{n, \text { target }}\right)^{2}
$$

where $C$ is an appropriately chosen coefficient. Currently the only parameter that is subject to this penalty is the convective velocity $U_{c}$.

At present, four functional forms for $p_{0}(x)$ have been examined:

(a) tanh-tanh

$$
p_{0}(x)=\tanh \left(x / b_{1}\right)^{p_{1}}\left\{1-\tanh \left(x / b_{2}\right)^{p_{2}}\right\} e^{i \alpha x}
$$

(b) $\mathrm{x}$-exp

(c) $\sin (\tanh )$

$$
p_{0}(x)=\left(x / b_{1}\right)^{p_{1}} e^{-\left(x / b_{2}\right)^{p_{2}}} e^{i \alpha x}
$$

$p_{0}(x)=\sin ^{p_{2}}\left[\pi \tanh \left(x / b_{1}\right)^{p_{1}}\right] e^{i \alpha x}$

(d) asymmetric “Gaussian”

$$
p_{0}(x)=\left\{\begin{array}{ll}
\exp \left(-\left|\frac{x-b_{1}}{b_{1}}\right|^{p_{1}}\right), & x \leq b_{1} \\
\exp \left(-\left|\frac{x-b_{1}}{b_{2}}\right|^{p_{2}}\right), & x>b_{1}
\end{array}\right\} e^{i \alpha x}
$$

All four functions involve an amplifying part and a decaying part. In (a), (c), and (d), the amplification is controlled by the length scale $b_{1}$ and power $p_{1}$, and the decay is controlled by length scale $b_{2}$ and power $p_{2}$. For function (c) the amplification-decay is governed by the sine term and the parameters $b_{1}$ and $p_{1}$ control the shape of the envelope. The first three functions start at $x=0$, which appears physically sensible given that no flow exists for $x<0$, while the last function (d) does not have a specific origin. Best 
functions so far are deemed (a) and (b), with some preference for (a) because it gives more independent control over the amplification and decay parts.

The noise source parameter vector is defined as:

$$
\begin{aligned}
& A_{1}=\frac{U_{c}}{U_{j}}=\frac{\omega / \alpha}{U_{j}} \\
& A_{2}=b_{1} \\
& A_{3}=b_{2} \\
& A_{4}=p_{1} \\
& A_{5}=p_{2} \\
& A_{6}=Q
\end{aligned}
$$

Constraints are placed on $A_{1}$ so that the convective velocity $U_{c}$ is close to a target value of about $0.5 U_{j}$. The wavenumber $\alpha$ is thus determined from the parameter $A_{1}$. The minimization process of Eq.13 uses the Restarted Conjugate Gradient method of Shanno and Phua ${ }^{11}$ (ACM TOM Algorithm 500). The cost function converged to 0.03 or less.

\section{Alignment}

The wavepacket parameterization based on far-field autospectra does not provide sufficient information for locating the axial position of the wavepacket relative to the nozzle. In other words, the coordinate system used in the definition of the functions in Eqs. 14a-d may not coincide with the nozzle coordinate system. To locate the wavepacket, we use far-field phased array results that provide the distribution of the noise source on the axial distance-frequency plane, and specifically the axial location of the peak source versus frequency. It is important to realize that far-field phased array measurements detect only the radiating part of the noise source. Consequently, the wavepacket is aligned so that the peak of the supersonic envelope $p_{0, \text { sup }}(x)$ coincides with the location of peak noise source as measured by the phased array. The alignment process is illustrated in Fig.5. For given frequency, the location of peak noise relative to the nozzle exit is $X_{0}$. The nozzle exit is located at distance $X_{\mathrm{te}}$ from the trailing edge of the shield. It is evident from the figure that the axial shield length, in the wavepacket frame of reference, is $x_{s}=X_{\mathrm{te}}-X_{0}+x_{\text {peak }}$. The vertical position of the shield, $y_{s}$, is defined relative to the wavepacket (jet) axis. Note that the Cartesian $(x, y, z)$ system used in the diffraction computation is the wavepacket coordinate system. The monopole was added at the location of the peak of the supersonic envelope, $x=x_{\text {peak }}$.

Some issues with this alignment procedure should be noted. First, conventional phased array imaging treats the noise source as a line of incoherent monopoles, which may be seen as incompatible with the current wavepacket modeling; second, refraction effects on the propagation of rays from the source to the microphone are typically not accounted for when processing phased array data. These limitations should be kept in mind as we refine our methods for properly positioning the wavepacket. 

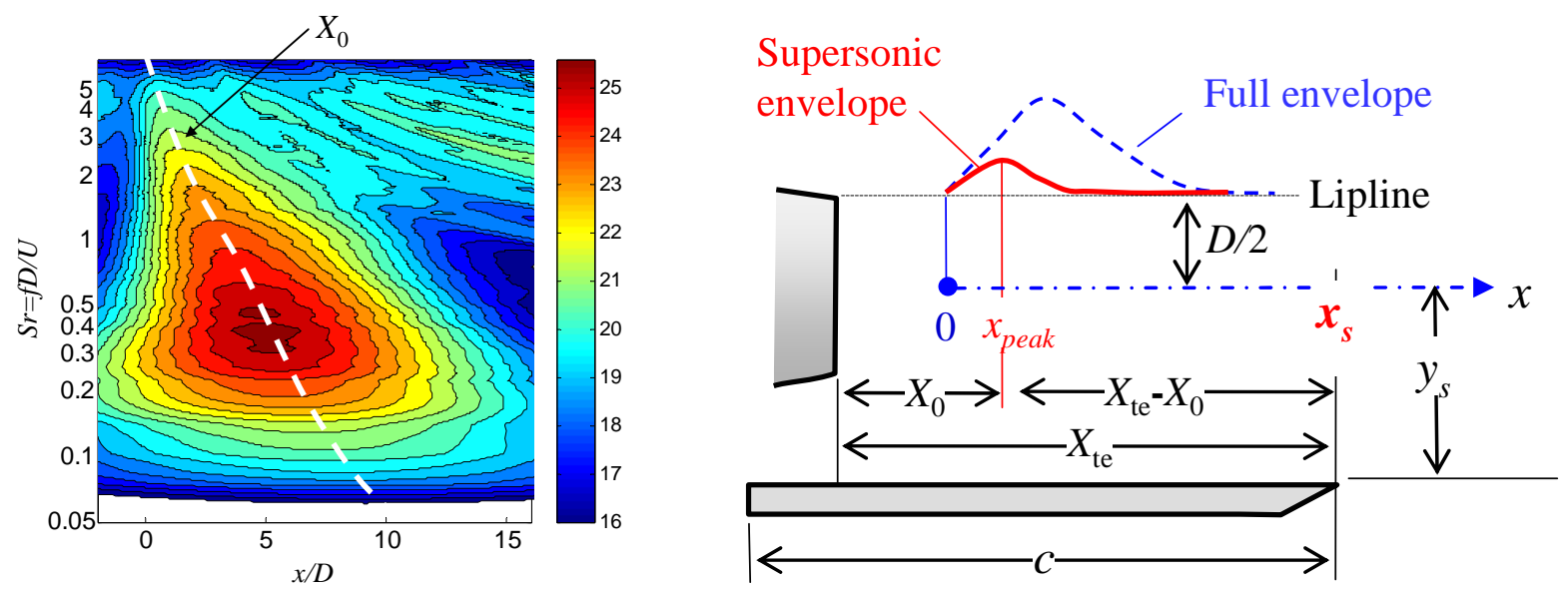

Fig. 5 Axial positioning of the wavepacket. Left: noise source map for $M=0.9$ cold jet; right: alignment of wavepacket based on peak of supersonic (radiating) envelope.

\section{Diffraction Predictions Using the Boundary Element Method}

\section{A. General overview of BEM}

The application and validation of the boundary element method (BEM) to various types of diffraction problems has been covered extensively in a previous publication ${ }^{4}$, so this section presents only the basic aspects of the method and its implementation. Figure 6 shows the basic setup for the shielding prediction discussed here. The boundary surface $S$ is the rectangular shield of Fig. 1 . The incident field $p_{i}$ comes from the modeled source discussed in the previous section. We seek the solution for the scattered field, $p_{s}$, at the field points $F$. The total field is then obtained by adding the incident and scattered fields. The BEM method solves the Helmholtz equation

$$
\nabla^{2} p+k^{2} p=0
$$

where $k=\omega / a_{\infty}$ is the wave number and $p=p(\mathbf{x}, \omega)$ is the complex pressure in the frequency domain. The solution to the Helmholtz equation is the boundary integral equation ${ }^{14}$,

$$
C(F) p(F)=-\int_{S}\left(i \rho \omega u_{n}(B) \psi+p(B) \frac{\partial \psi}{\partial n}\right) d S+p_{i}
$$

where $\psi$ is the solution of the Helmholtz equation in 3D free space,

$$
\psi=\frac{1}{4 \pi r} e^{-i k r}
$$

and $r$ denotes here the distance from the field point $F$ to the surface point $B$. In Eq.17, the factor $C$ takes different values depending on whether the field point $F$ is located in the acoustic domain, or in the interior of the object, or on the object surface $S$,

\section{B. Codes}

$$
C(F)= \begin{cases}1, & F \text { in acoustic domain } \\ 1-\int_{S} \frac{\partial}{\partial n}\left(\frac{1}{4 \pi R}\right) d S, & F \text { on object surface } \\ 0, & F \text { in object interior }\end{cases}
$$

Two BEM codes were investigated: a conventional method by $\mathrm{Wu}^{12}$, modified by us to allow for a userdefined incident field; and a Fast Multiple version (FastBEM, CAE Research Lab), developed by Liu ${ }^{13}$. The conventional method generates an exact solution but is limited to matrix sizes of up to about 
$10000 \times 10000$ elements. This means that the boundary surface cannot have more than $10^{4}$ elements, which in our case limits us to Strouhal numbers on the order of 0.5. FastBEM can handle much larger matrices, possibly up to $10^{5}$ boundary elements. It generates an approximate solution based on an iterative algorithm with a specified tolerance, set here at 0.001. The fast-multipole solution is sensitive to the multipole expansion level, which is supposed to scale according to the non-dimensional wavenumber $k L$, with $L$ the largest size of the boundary (in our case usually the span of the shield), but at a significant computational cost. Extensive comparisons, at low to mid frequencies, were conducted between conventional and fast-multipole BEM for problems involving diffraction of monopoles and wavepackets. Practically identical solutions were obtained, providing confidence for the application of FastBEM to the current problem. The boundary surface was discretized using triangular meshes with a resolution of 8 nodes per wavelength. For $S r=1.0$ the number of elements was approximately 25000.

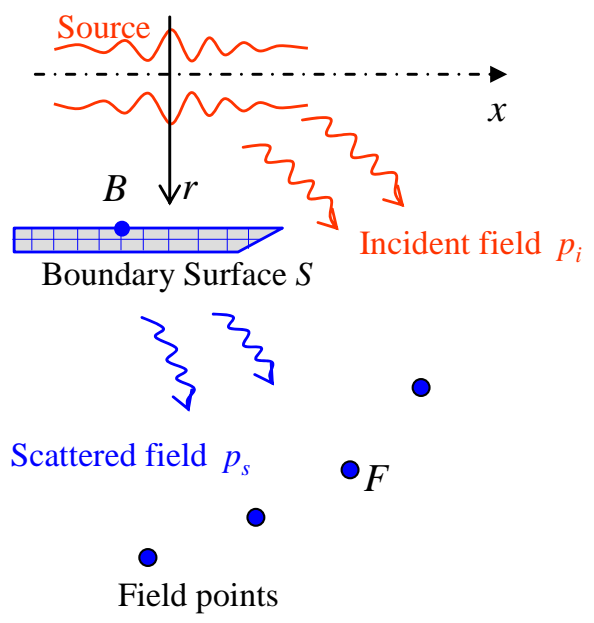

Fig. 6 Setup for the Boundary Element Method (BEM).

\section{Integration of results}

As described in Section III, the incident field is created by the incoherent addition of two sources: the wavepacket and the monopole. This means that the BEM must be run for each individual incident field, then the intensities (spectra) are summed. A further consideration is that for non-axisymmetric sources (azimuthal modes $m \neq 0$ ), the total field is asymmetric around the $z=0$ plane (Fig.1). In a real application, however, the intensity (autospectrum) of the total field must be symmetric around the $z=0$ plane. This requires computation of the anti-symmetric problem, with azimuthal mode $-m$, under the assumption that azimuthal modes $m$ and $-m$ are uncorrelated. We then average the intensities of the total fields arising from each mode. A related observation is that for azimuthal modes $m \neq 0$ the BEM computation cannot benefit from reduction to a half-space problem.

In summary, the diffraction computation entails the following steps:

- $\quad$ BEM solution for wavepacket incident field $p_{\mathrm{w}}(m, r, x, \phi, t)$, given by Eq. 5 . The resulting intensity of the total field is $S_{T, w}(m, x, y, z)$, with $y=-r \cos \phi$ and $z=r \sin \phi$.

- $\quad$ BEM solution for wavepacket incident field $p_{\mathrm{w}}(-m, r, x, \phi, t)$, given by Eq. 5 . The resulting intensity of the total field is $S_{T, w}(-m, x, y, z)$. However, this is equivalent to $S_{T, w}(m, x, y,-z)$, so it does not require a new computation, provided that the field points are arranged symmetrically around the $z=0$ plane.

- BEM solution for monopole incident field $p_{p}(R, t)$, given by Eq.9. The resulting intensity of the total field is $S_{T, p}(x, y, z)$, with $R^{2}=x^{2}+y^{2}+z^{2}$. 
- Combination of the above intensity fields as follows:

$$
S_{T}(x, y, z)=\frac{1}{2}\left[S_{T, w}(m, x, y, z)+S_{T, w}(m, x, y,-z)\right]+S_{T, p}(x, y, z)
$$

\section{Results}

\section{A. Parameterization}

The jet in this investigation was cold with Mach number $M_{j}=0.9$, velocity $U_{j}=285 \mathrm{~m} / \mathrm{s}$, and diameter $D_{j}=21.8 \mathrm{~mm}$. Parameterization of the wavepacket requires judicious choices for the azimuthal mode and a reasonable constraint for the convective velocity ratio $U_{c} / U_{j}$. Experimentation with the minimization process of Eq. 13 shows that, for given frequency, there are one or two azimuthal modes that can fit the experimental results very well, while for other modes it is impossible to get a good fit regardless of the parameter values. For example, for $S r=0.2$ modes $m=0$ and 1 work best; for $S r=0.5, m=2$; and for $S r=1.0$, $m=2$. The predominance of modes $m=0,1,2$ is in line with results of azimuthal microphone measurements on similar jets by Juve et al. ${ }^{14}$ and by Brown and Bridges ${ }^{15}$. The other constraint is the convective velocity ratio $U_{c} / U_{j}$, which here is set to range between 0.45 (low frequency) to 0.6 (high frequency). Figure 7 shows parameterizations results for Strouhal numbers $S r=0.2,0.5$, and 1.0. The "tanh-tanh" wavepacket function of Eq. 14a was used. Plotted are the full wavepacket shape $p_{0}(x)$, the supersonic portion of $p_{0}(x)$, and the modeled and experimental intensity distributions. Note that the amplitude of the $\mathrm{p}_{0 \text {,sup }}(x)$ is about two orders of magnitude less than $p_{0}(x)$, underscoring how small a fraction of the initial perturbation radiates to the far field. There are significant differences between the shapes of $p_{0}(x)$ and $\mathrm{p}_{0 \text {,sup }}(x)$ owing to the truncation of the Fourier transform in Eq. 5b, manifested by the sinc convolution of Eq. 6. Figure 7 demonstrates that good fits can be obtained between the modeled and experimental intensity polar distributions, with errors of less then 3\% in the cost function of Eq. 13.

(a)
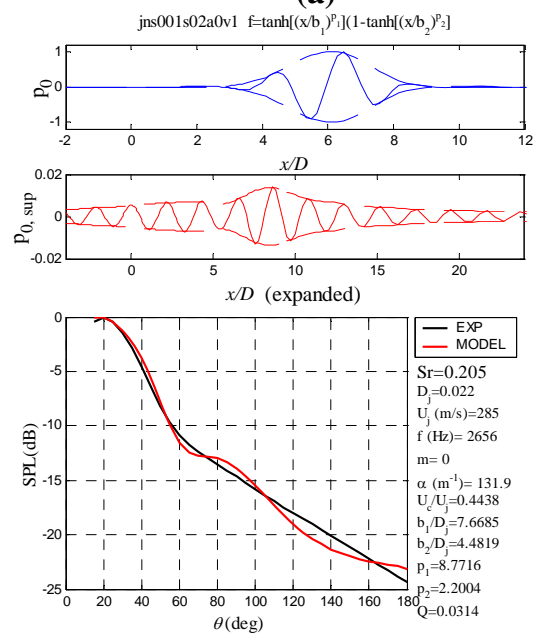

(b)
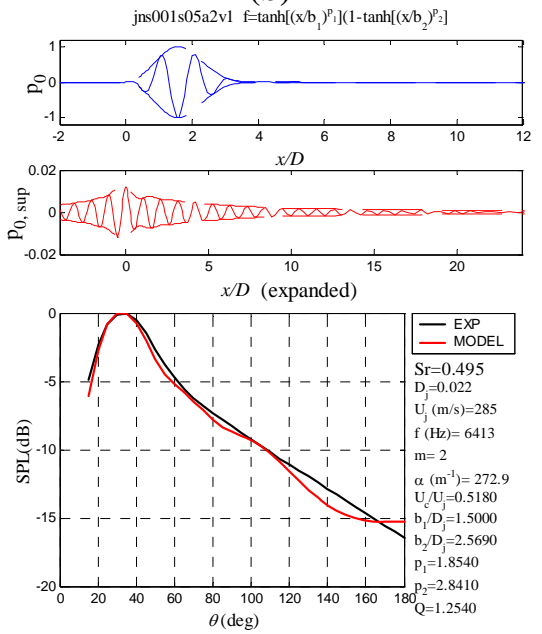

(c)
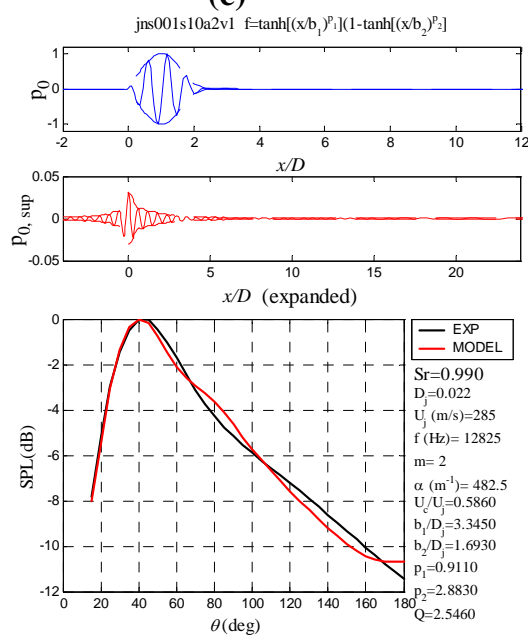

Fig. 7 Results of parameterization for (a) $\mathrm{Sr}=0.2$; (b) $\mathrm{Sr}=0.5$, and (c) $\mathrm{Sr}=1.0$ 


\section{B. Diffraction predictions}

Results are presented for the generic geometry of Fig.1 with the following boundary dimensions: chord lenth $c=178 \mathrm{~mm}$; span $s=600 \mathrm{~mm}$; thickness $t=15 \mathrm{~mm}$; trailing edge angle $=30^{\circ}$; and distance between nozzle exit and trailing edge $X_{t e}=102 \mathrm{~mm}$. These are the boundary dimensions used in the experiments, except that the plate thickness in the experiments was $3.2 \mathrm{~mm}$. The BEM cannot handle very thin plates, so the plate is thicker for the computations. Trials with different thicknesses showed insensitivity of the results for $0.05<t / c<0.2$. The same holds true for trailing edge angles above $20^{\circ}$. The parameters listed in Fig. 7 were used to model the noise source.

We begin the presentation of results with plots of the intensity distributions on a downward polar arc $\left(\phi=0^{\circ}\right)$ and a sideline polar arc $\left(\phi=60^{\circ}\right)$, both arcs having a radius $R=1 \mathrm{~m}$. The distributions are plotted versus polar angle $\theta$ for a given Strouhal number and are compared to the experimental data. Note that the experimental data are limited to $\theta=120^{\circ}$. Figures 8, 9, and 10 present such plots for Strouhal numbers $S r=0.2,0.5$, and 1.0, respectively. For $S r=0.2$ the computations capture the noise excess created by the boundary, as seen in the experimental spectra of Fig.1. For $S r=0.5$ and 1.0, the boundary reduces noise and the model captures the experimental trends reasonably well, with some overprediction of the reduction at $S r=1.0$. At large polar angles we observe oscillations of the modeled intensity, particularly at $S r=1.0$. This is the result of interference caused by the deterministic nature of the noise source model used.
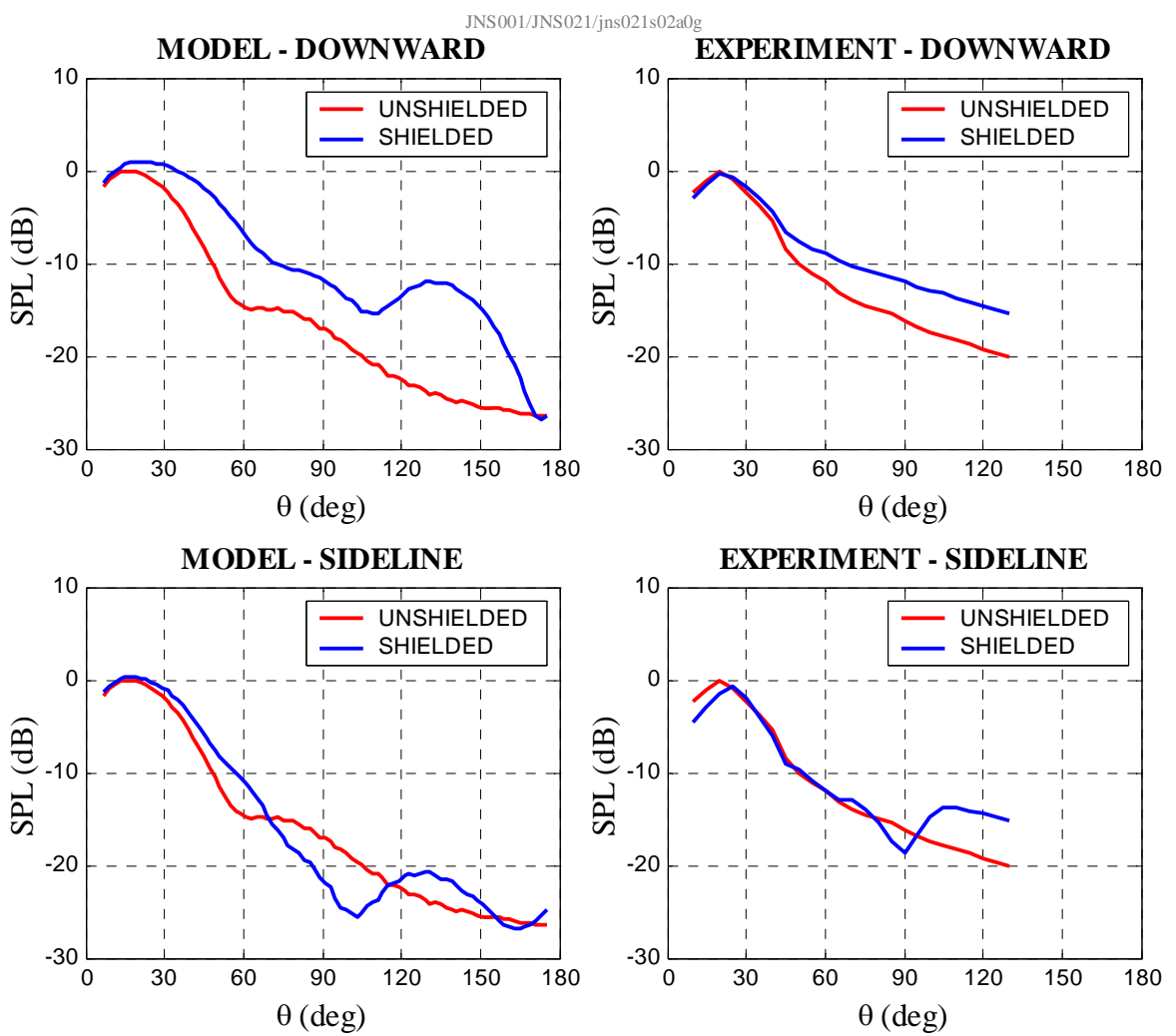

Fig. 8 Intensity distributions on downward and sideline arcs for $\mathrm{Sr}=\mathbf{0 . 2}$. 

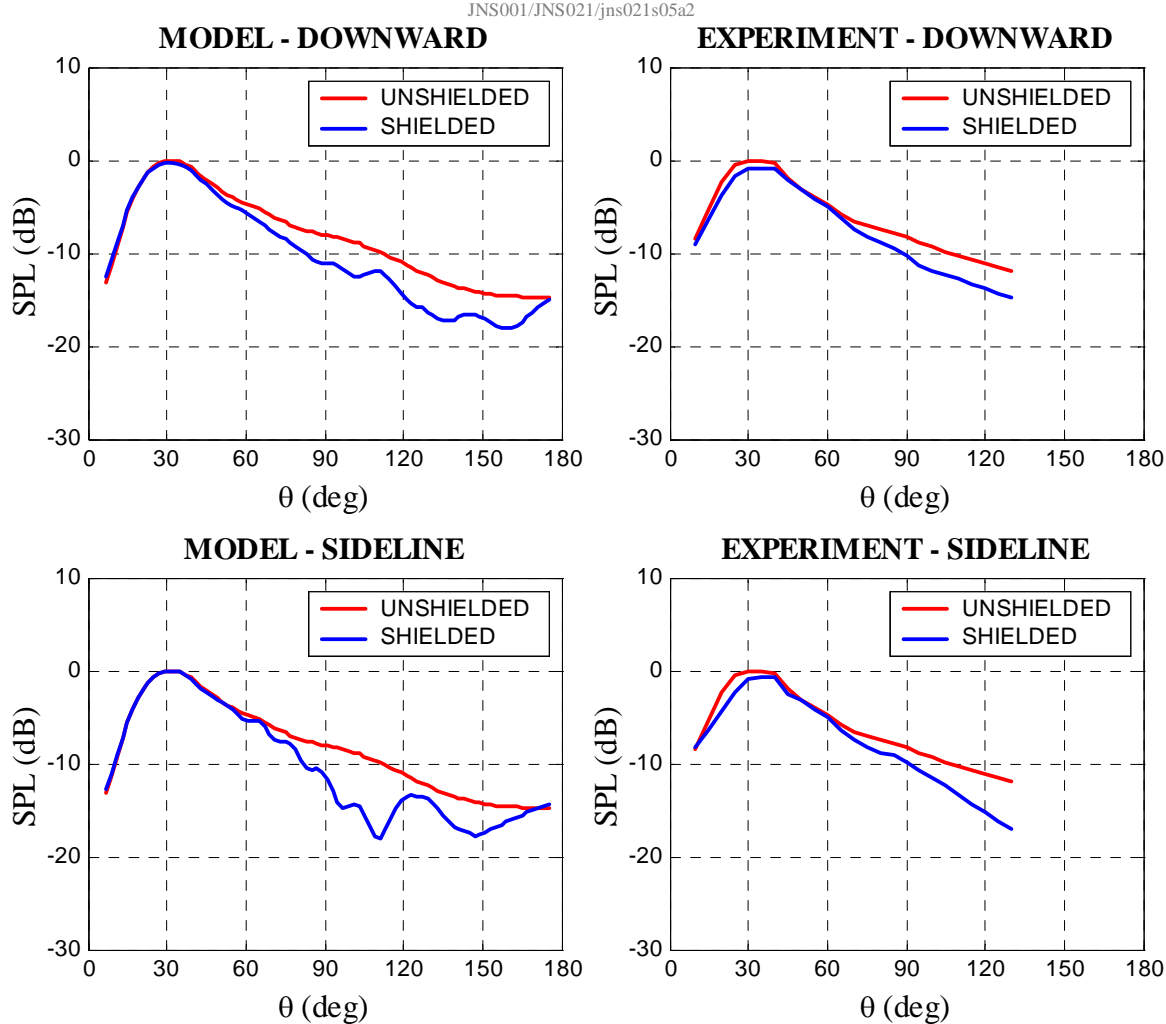

Fig. 9 Intensity distributions on downward and sideline arcs for $\mathrm{Sr}=\mathbf{0 . 5}$
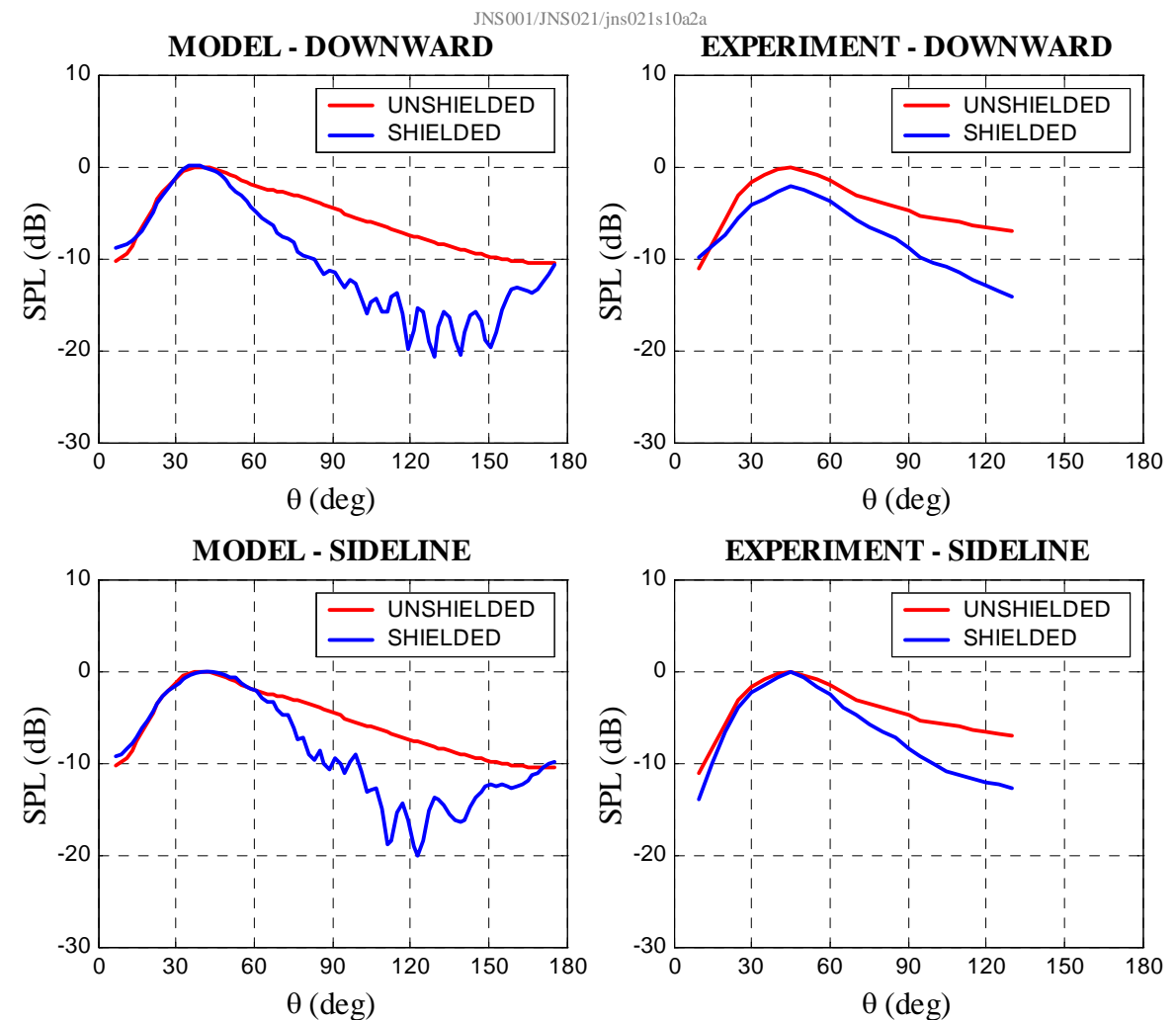

Fig. 10 Intensity distributions on downward and sideline arcs for $S r=1.0$ 
Figure 11 shows the relative contributions of the wavepacket and monopole to the diffraction problem for $\mathrm{Sr}=0.5$. The insertion loss for the monopole is much greater than that of the wavepacket. As a result, the total (incident plus scattered) intensity field for the combined source is practically equal to that of the wavepacket alone, except near $\theta=180$ deg. For aircraft noise, only angles up to $\theta=150$ deg are important. Therefore, acceptable solutions may be generated by computing only the diffraction of the wavepacket (thus saving 50\% of the computational cost), and using the incident field based on the combination of the wavepacket and monopole for computing the insertion loss.

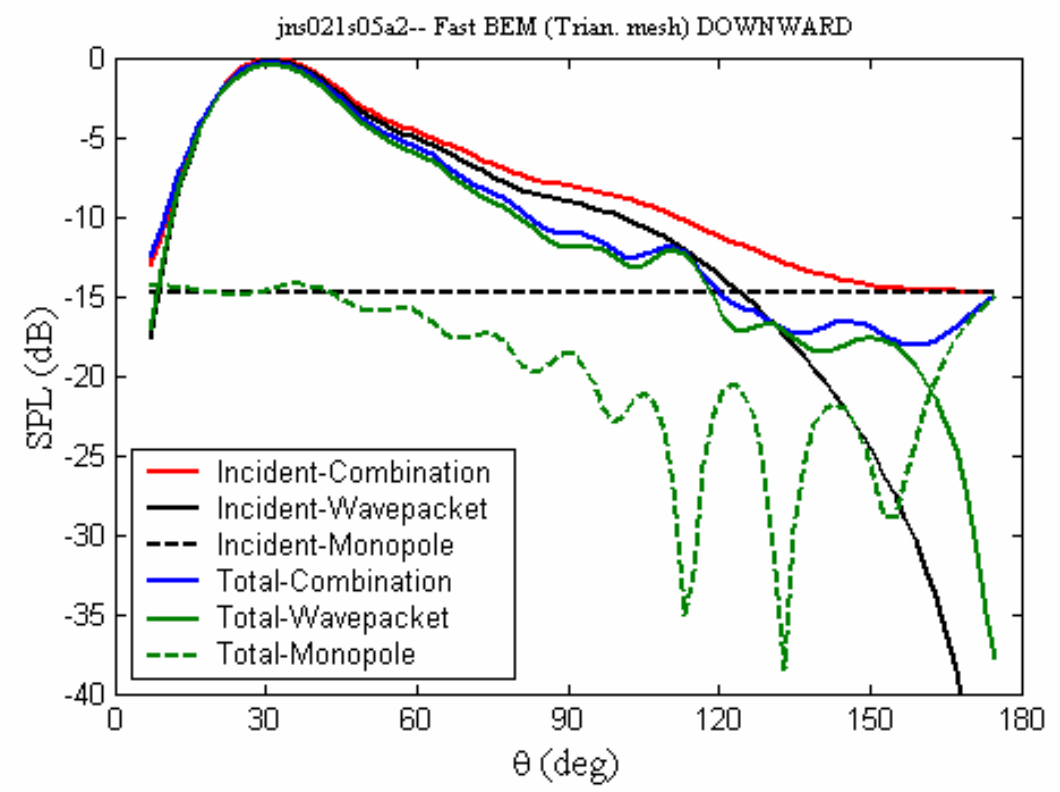

Fig. 11 Relative contributions of wavepacket and monopole sources to the incident and total fields for $\mathrm{Sr}=0.5$

Further insight into the physics of diffraction is gained by examining contours of the incident and total intensity fields on the symmetry plane $z=0$ in the near field. These are presented in Figs. 12, 13, and 14 for $S r=0.2,0.5$, and 1.0 , respectively. White dots indicate the nodes of the boundary on the symmetry plane. For $S r=0.2$, the generation of the excess noise is evident in the contour plots showing strong diffraction of sound towards large polar angles as the shield penetrates more into the acoustic field created by the wavepacket. For $S r=0.5$ and 1.0, there is clear attenuation of the total intensity field as the polar angle increases.

Finally, Fig. 15 presents contours of the insertion loss on the "ground" plane $y=-1 \mathrm{~m}$ for $S r=0.5$. The source center is indicated at the red circle, and the white region represents the boundary. It is seen that the insertion loss peaks in the general direction of maximum distance between source and edge of the boundary. 

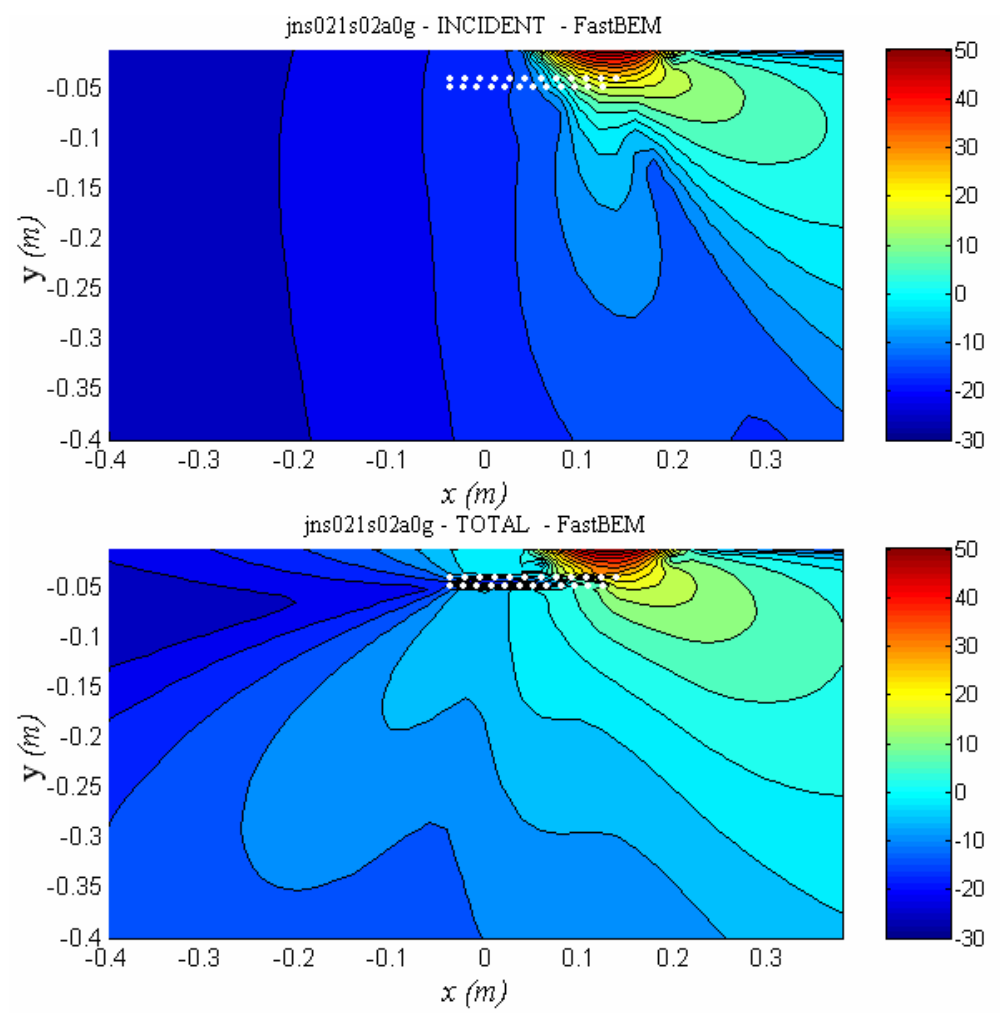

Fig. 12 Decibel contours of incident (top) and total (bottom) pressure fields on symmetry plane for $\mathrm{Sr}=0.2$.
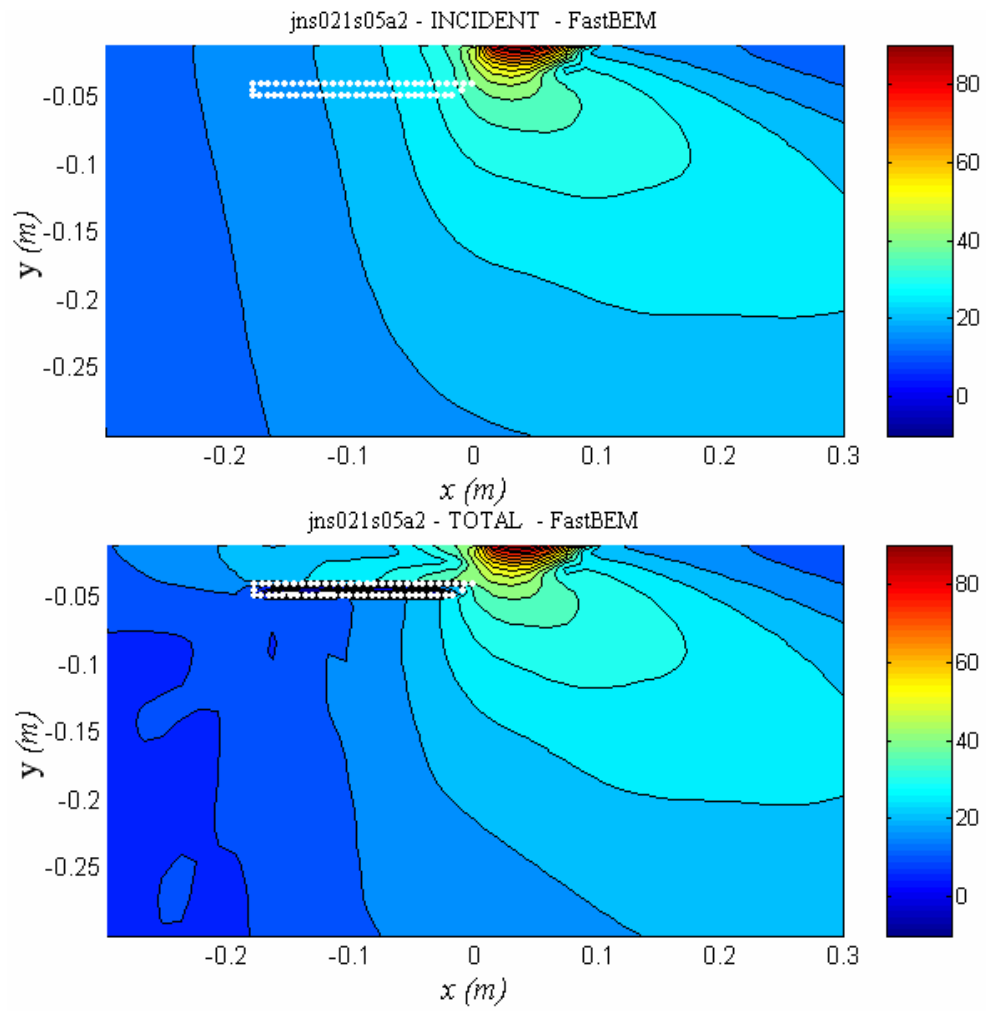

Fig. 13 Decibel contours of incident (top) and total (bottom) pressure fields on symmetry plane for $\mathrm{Sr}=0.5$. 

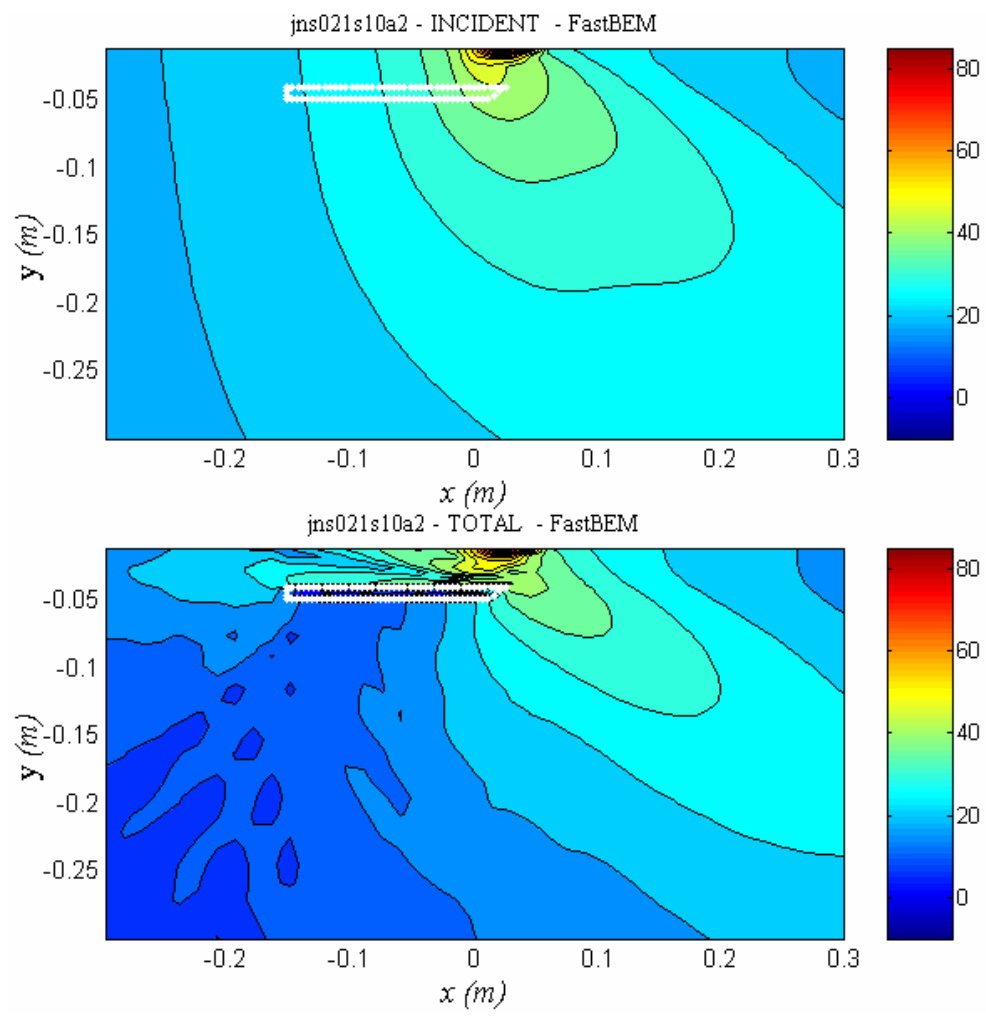

Fig. 14 Decibel contours of incident (top) and total (bottom) pressure fields on symmetry plane for $S r=1.0$.

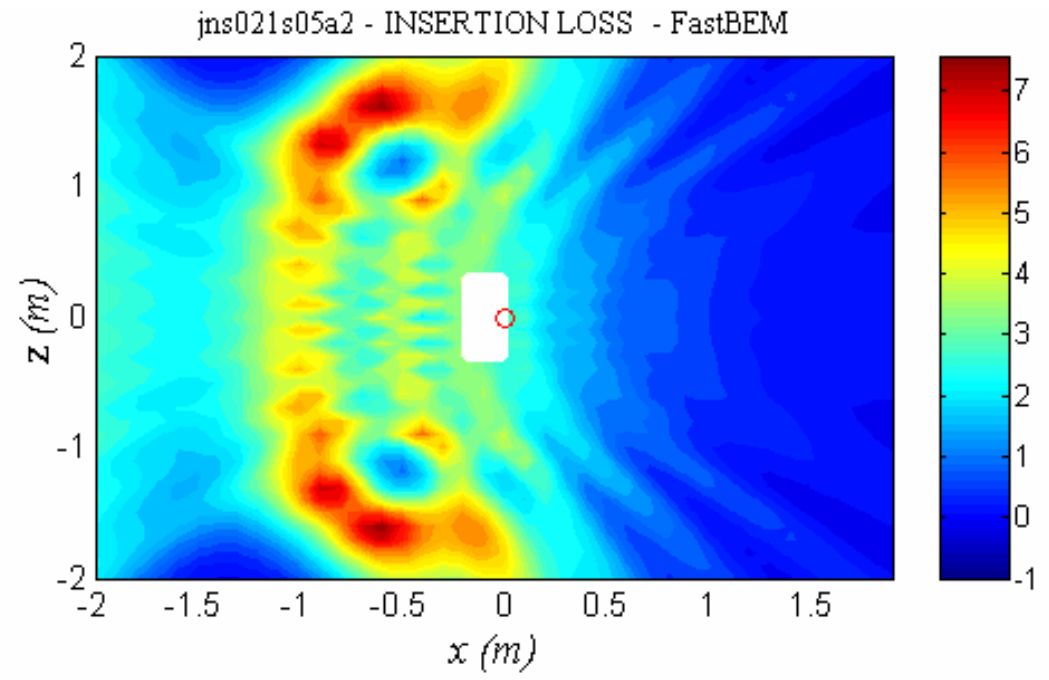

Fig. 15 Contours of insertion loss $(\mathrm{dB})$ on the plane $\mathrm{y}=-1 \mathrm{~m}$ for $S r=0.5$.

\section{Conclusions}

In an effort to increase the fidelity of predictive methods for diffraction of jet noise, the jet noise source was modeled as an incoherent superposition of a wavepacket and a monopole. The modeling was applied to a Mach 0.9 jet shielded by a rectangular plate. The source model parameters were determined by minimizing the difference between modeled and experimental far-field polar intensity distributions for the free jet. Constraints on the convective velocity help guide the minimization towards physical outcomes. Very good agreement is obtained between the modeled and experimental distributions for the incident intensity field. 
Computation of the diffraction used the boundary element method (BEM) in fast-multipole implementation. Results of the computation were compared with experimental intensity distributions for Strouhal numbers 0.2, 0.5, and 1.0. Reasonable agreement is found and the computation captures specific features of diffraction such as amplification of sound at low frequency. This indicates that the wavepacket incident field contains the salient features for jet noise diffraction. The monopole had minor contribution to the total field, so the diffraction was influenced mainly by the wavepacket source.

A fundamental limitation of the current approach is that it uses a deterministic noise source to model a phenomenon that is inherently random. Manifestations of this limitation are seen in the oscillations of the intensity (Fig.10, for example) arising from the coherent interaction of diffracted ray paths. The problem is not serious for the frequencies examined, but may become severe at higher frequency. The use of a deterministic model for the noise source is necessitated by the nature of the BEM. The diffraction of a random source should ideally be addressed by Stochastic BEM, a tool that is not yet available in a useful form.

\section{Acknowledgment}

This research has been funded by Boeing Subcontract No. 208547 in support of NASA contract NNL07AA54C "Acoustic Prediction Methodology and Test Validation for an Efficient Low-Noise Hybrid Wing Body Subsonic Transport.” The author thanks Prof. Y.J. Liu, University of Cincinnati, for creating the option of a user-defined incident field in the FastBEM code.

\section{References}

${ }^{1}$ Von Glahn, U., Groesbeck, D., and Reshotko, M. "Geometry Considerations for Jet Noise Shielding with CTOL Engine-Over-The-Wing Concept,” AIAA Paper 74-568, June 1974.

${ }^{2}$ Russell, J., and Berton, J., "Stone Jet Noise Module (ST2JET)", ANOPP Theoretical Manual, ver.25, NASA Langley Research Center, Hampton, VA, 2006.

${ }^{3}$ Maekawa, Z., “Noise Reduction by Screens,” Applied Acoustics, Vol.1, 1968, pp. 157-173.

${ }^{4}$ Huang, C., and Papamoschou, D., "Numerical Study of Noise Shielding by Airframe Surfaces," AIAA-20082999, May 2008.

${ }^{5}$ Tam, C.K.W., Golebiowski, M., and Seiner, J.M., "On the Two Components of Turbulent Mixing Noise from Supersonic Jets,” AIAA Paper 96-1716, Jan..1996.

${ }^{6}$ Tam, C. K. W., and Burton, D. E., "Sound Generation by the Instability Waves of Supersonic Flows. Part 2.Axisymmetric Jets," Journal of Fluid Mechanics, Vol. 138, 1984, pp. 273-295.

${ }^{7}$ Crighton, D.G. and Huerre, P., "Shear-Layer Pressure Fluctuations and Superdirective Acoustic Sources," Journal of Fluid Mechanics, Vol. 220, 1990, pp. 355-368.

${ }^{8}$ Avital, E.J., Sandham, N.D., and Luo, K.H., "Mach Wave Radiation by Mixing Layers. Part I: Analysis of the Sound Field,” Theoretical and Computational Fluid Dynamics, Vol. 12, 1998, pp. 73-90.

${ }^{9}$ Morris, P.J., "Jet Noise Prediction: Past Present and Future," Canadian Acoustics, Vol. 35, No. 3, September 2007, pp. 16-22.

${ }^{10}$ Reba, J., Simonich, J., and Schlinker, R., "Sound Radiated by Large-Scale Wave-Packets in Subsonic and Supersonic Jets,” AIAA Paper 2009-3256, May 2009.

${ }^{11}$ Shanno, D.F., and Phua, K.H., "Minimization of Unconstrained Multivariate Functions," ACM Transactions on Mathematical Software, Vol. 6, pp. 618-622.

${ }^{12} \mathrm{Wu}, \mathrm{T} . \mathrm{W} ., \mathrm{Boundary}$ Element Acoustics, WIT Press, Boston, 2000.

${ }^{13}$ Liu, Y.J., Fast Multipole Boundary Element Method - Theory and Applications in Engineering, Cambridge University Press, Cambridge, 2009.

${ }^{14} J u v e$, D., Sunyach, M., and Comte-Bellot, G., "Filtered Azimuthal Correlations in the Acoustic Far Field of a Subsonic Jet,” AIAA Journal, Vol. 17, No.1, 1979, pp. 112-113.

${ }^{15}$ Brown, C., and Bridges, J., "Acoustic Efficiency of Azimuthal Modes in Jet Noise Using Chevron Nozzles, AIAA Paper 2006-2654, Jan. 2006. 